\title{
Chemotherapy within 30 days before surgery does not augment postoperative mortality and morbidity
}

\section{La chimiothérapie dans un délai de 30 jours avant l'intervention chirurgicale n'augmente pas la mortalité et la morbidité postopératoires}

\author{
Alparslan Turan, MD • Diana Shao - Vafi Salmasi, MD • Hooman Honar, MD • \\ Abdulkadir Atim, MD • Jarrod E. Dalton, MA • Leif Saager, MD • \\ Daniel I. Sessler, MD
}

Received: 10 January 2012/ Accepted: 8 May 2012/Published online: 26 May 2012

(C) Canadian Anesthesiologists' Society 2012

\begin{abstract}
Background Preoperative chemotherapy is frequently given to shrink or decrease the chance of metastasis. However, chemotherapy has well-recognized side effects that may complicate the perioperative period. We therefore tested the hypotheses that chemotherapy within 30 days before cancer surgery is associated with an increased risk of mortality and with a composite of major morbidities within 30 postoperative days.

Methods We evaluated 971,455 patients from the American College of Surgeons National Surgical Quality Improvement Program database. Patients were defined as having chemotherapy when they were given any chemotherapy for malignancy within 30 days before surgery. We
\end{abstract}

\begin{abstract}
Author contributions Alparslan Turan, Jarrod E. Dalton, Leif Saager, and Daniel I. Sessler contributed to the conception and design of the study. Diana Shao, Vafi Salmasi, Hooman Honar, Abdulkadir Atim, Jarrod E. Dalton, and Daniel I. Sessler contributed to the acquisition of data. Alparslan Turan, Diana Shao, Jarrod E. Dalton, and Daniel I. Sessler contributed to the analysis and interpretation of data. Alparslan Turan, Diana Shao, Vafi Salmasi, Hooman Honar, Abdulkadir Atim, Jarrod E. Dalton, Leif Saager, and Daniel I. Sessler participated in drafting the article. Alparslan Turan, Vafi Salmasi, Hooman Honar, Abdulkadir Atim, Jarrod E. Dalton, Leif Saager, and Daniel I. Sessler contributed to revising the article.
\end{abstract}

A. Turan, MD $(\varangle) \cdot$ D. Shao · V. Salmasi, MD · H. Honar, MD · A. Atim, MD - L. Saager, MD - D. I. Sessler, MD Department of Outcomes Research, Cleveland Clinic, 9500 Euclid Avenue, P-77, Cleveland, OH 44195, USA

e-mail: alparslanturan@yahoo.com

URL: www.OR.org

J. E. Dalton, MA

Departments of Quantitative Health Sciences and Outcomes Research, Cleveland Clinic, Cleveland, OH, USA successfully matched 1,348 pairs of chemotherapy recipients and non-recipients.

Results Twenty-one of the 1,348 (1.6\%) non-chemotherapy patients died within 30 days after surgery compared with 30 of the $1,348(2.2 \%)$ chemotherapy patients. The odds of mortality were not statistically different between groups based on our logistic regression model [odds ratio $(O R)=1.47 ; 95 \%$ confidence interval $(C I) 0.82$ to 2.64; $P=0.19]$. The most common complication observed was wound infection in $13.1 \%$ of non-chemotherapy patients compared with $14.2 \%$ of the chemotherapy patients. There was similarly no difference between groups for the collapsed composite of major morbidities [OR $=1.17 ; 95 \%$ CI 0.97 to $1.42 ; P=0.09]$.

Conclusion Preoperative use of neoadjuvant chemotherapy in cancer patients undergoing resection surgeries was not associated with a higher rate of early postoperative complications or mortality.

Résumé

Contexte La chimiothérapie préopératoire est souvent utilisée pour réduire la taille de métastases ou en diminuer le risque. Cependant, la chimiothérapie entraîne des effets indésirables bien identifiés qui risquent de compliquer la période périopératoire. Nous avons donc testé les hypothèses selon lesquelles la chimiothérapie administrée dans un delai de 30 jours avant une chirurgie oncologique était associée à une augmentation du risque de mortalité et à un ensemble de morbidités majeures survenant dans les 30 premiers jours postopératoires.

Méthode Nous avons évalué 971455 patients de la base de données du Programme national pour l'amelioration de la qualité de la chirurgie de l'American College of 
Surgeons. Les patients étaient considérés avoir eu une chimiothérapie quand ils en avaient reçu une pour tumeur maligne dans les 30 jours précédant l'intervention chirurgicale. Nous avons apparié avec succès 1348 paires de patients (patients ayant reçu une chimiothérapie et patients n'en ayant pas reçu).

Résultats Parmi les 1348 patients n'ayant pas reçu de chimiothérapie, 21 (1,6\%) sont décédés dans les 30 jours suivant l'intervention, comparativement à 30 (2,2\%) des 1348 patients ayant reçu une chimiothérapie. La probabilité de mortalité n'a pas été statistiquement différente entre les deux groupes d'après notre modèle de régression logistique (rapport de cotes $[R C]=1,47$; intervalle de confiance à 95 \% [IC] : 0,82 à 2,64; $P=0,19)$. La complication observée la plus fréquente a été une infection de la cicatrice chez. 13,1\% des patients n'ayant pas reçu de chimiothérapie et $14,2 \%$ des patients ayant reçu une chimiothérapie. De même, il n'y a pas eu de différence entre les groupes pour l'ensemble des morbidités majeures $(R C=1,17 ; 95 \%$ IC : 0,97 à 1,42; $P=0,09$ ).

Conclusion L'administration préopératoire d'une chimiothérapie néoadjuvante chez des patients atteints de cancer et devant subir une excision chirurgicale n'a pas été associée à des taux de complications postopératoires précoces ou de mortalité plus élevés.

Cancer is the second leading cause of death in the United States killing more than 500,000 Americans in 2009. ${ }^{1}$ Surgery, radiation, and chemotherapy are the mainstays of cancer treatment. Chemotherapy can be used as a primary treatment or as an adjunct to surgery with or without concomitant radiation. Chemotherapy is sometimes used before surgery — as a neoadjuvant treatment — or after surgery, often for more advanced cancer.

Neoadjuvant chemotherapy is generally used when it is expected to shrink tumours and thus facilitate surgery or when it might reduce the risk of local or metastatic cancer recurrence. ${ }^{2}$ On the other hand, chemotherapy also causes cardiac toxicity, pulmonary damage, and myelosuppression, with consequent anemia, coagulopathy, and infection risk. The anti-cancer benefits of neoadjuvant chemotherapy are thus balanced by the potential acute perioperative risk induced by these relatively toxic drugs.

Meta-analyses of randomized trials along with subsequent randomized trials suggest that neoadjuvant chemotherapy and/or radiation therapy slightly increases the risk of morbidity and mortality. ${ }^{3-6}$ Nordlinger et al. ${ }^{7}$ evaluated the effect of perioperative chemotherapy on hepatic metastasis and showed a $36 \%$ increase in complications with chemotherapy. Other studies, though, failed to show any association between preoperative chemotherapy and increased postoperative mortality or morbidity. ${ }^{8-12}$
The results of available studies are thus inconsistent; furthermore, they suffer from limited sample size and the fact that most were restricted to specific types of cancer. More inclusive studies with larger sample sizes are therefore warranted. We consequently tested the hypotheses that cancer chemotherapy within 30 days before surgery is associated with increased risk of 1) 30-day postoperative mortality; and, 2) a composite of major morbidities within 30 postoperative days.

\section{Methods}

With the approval of the Institutional Review Board, we analyzed patients enrolled in the American College of Surgeons National Surgical Quality Improvement Program (ACS-NSQIP) registry from January 1, 2005 to December 31, 2009. A dedicated clinical nurse reviewer collected data from patients' medical records, physicians' office records, and telephone interviews with patients. The data were collected prospectively using standardized definitions of each variable and outcome. The accuracy and reproducibility of these data have been established. ${ }^{13,14}$

\section{Data sources}

Patients were identified by interrogating the ACS-NSQIP database for Current Procedural Terminology (CPT) codes relating to cancer surgery (Table 1). Patients were subsequently excluded if the extracted ACS-NSQIP record indicated: functionally dependent (patient's ability to perform activities of daily living in 30 days prior to surgery, dependent defined as the patient requiring total assistance for all activities of daily living), American Society of Anesthesiologists' physical status classification of "moribund" (patient who is not expected to survive without an operation), preoperative pneumonia (evidence of preoperative pneumonia determined by clinical, radiological and microbiological evidence), ventilator-assisted respiration during the $48 \mathrm{hr}$ before surgery, preoperative coma, openwound infection before surgery, or preoperative sepsis. In the ACS-NSQIP database, patients were defined as having

Table 1 Types of cancer and their associated CPT codes

\begin{tabular}{ll}
\hline Type of cancer & Associated CPT codes \\
\hline Breast Cancer & $19160,19162,19180,19240$ \\
Colon Cancer & $44320,44140,44141,44143,44145,44146$ \\
Rectal Cancer & 45110 \\
Liver Cancer & $47120,47122,47125,47130$ \\
Pancreatic Cancer & $48140,48150,48153$
\end{tabular}

CPT $=$ Current Procedural Terminology 
chemotherapy when they had any chemotherapy for malignancy within 30 days before surgery (hormonal therapy was not included).

Our primary outcomes were 30-day postoperative mortality and a collapsed composite (any $v s$ none) of the major morbid outcomes listed in Table 2, each of which is potentially related to chemotherapy-induced toxicity. Crude odds ratios (OR) comparing chemotherapy patients with those who were not given chemotherapy were estimated to describe population trends; however, these results were subject to potential confounding because the baseline characteristics differed.

To limit confounding, we matched chemotherapy patients to non-chemotherapy patients on both propensity scores and CPT code. The propensity scores were estimated using logistic regression with chemotherapy as the response and the baseline variables as the predictors (see Table 3). A 1:1 greedy matching algorithm was used with 0.01 propensity score units as the maximum allowable distance for matching. Exact matching on CPT code was enforced. To evaluate the success of matching, absolute standardized difference (ASD, or the absolute difference in means or proportions divided by the pooled standard deviation) scores were calculated for all unmatched patients and the subset of matched patients. Absolute standardized difference scores were used to summarize balance between the two samples. Although there is no consensus on what value of standardized difference denotes important residual imbalance, values of $<0.1$ are generally considered to represent reasonably balanced groups. ${ }^{15}$
Potential confounders displaying such imbalance were used for statistical adjustment in all subsequent analyses.

Matched chemotherapy patients and non-chemotherapy patients were compared on 30-day mortality and the collapsed composite outcome using respective multivariable conditional logistic regression models. Conditional logistic regression accounts for any correlation in outcomes that may be exhibited among patients within a given matched pair. Variables with a standardized difference of $>0.1$ were included in the model in an attempt to address any residual confounding. We used Wald's Chi square test for regression model parameters to evaluate the null hypothesis of no difference in outcomes between those who did and those who did not receive chemotherapy preoperatively. The type I error rate was set at 5\%. Odds ratios and 95\% confidence intervals (CI) were also estimated using the conditional logistic regression models.

Our collapsed composite outcome was defined as any or none of the major morbidities. However, because the major morbidity outcomes are likely not of equal weight, we performed a sensitivity analysis in which we weighted the major morbidity outcomes by clinical severity. First, three otherwise uninvolved anesthesiologists independently scored the relative severity of each individual outcome on a scale of $0-100$, with 100 being the most severe. The average of those scores for a given outcome was defined as a severity weight for that outcome. The weights are given in Table 4. A multivariate generalized estimating equations (GEE) model was then used to estimate a severity-weighted common effects OR across the components. ${ }^{16}$

Table 2 Definitions of the major complications analyzed in this study

\begin{tabular}{|c|c|c|c|}
\hline Complication & Definition & $\begin{array}{l}\text { Overall } \\
\text { Incidence }\end{array}$ & $\begin{array}{l}\text { Matched } \\
\text { Incidence }\end{array}$ \\
\hline 30-day mortality & postoperative 30 -day mortality & $1.7 \%$ & $1.9 \%$ \\
\hline Systemic infections & $\begin{array}{l}\text { systemic inflammatory response syndrome (SIRS), sepsis, } \\
\text { or septic shock }\end{array}$ & $7.2 \%$ & $7.0 \%$ \\
\hline Respiratory morbidity & $\begin{array}{l}\text { pneumonia, failure to wean from ventilation at } 48 \mathrm{hr} \\
\text { postoperative, or unplanned tracheal intubation }\end{array}$ & $5.2 \%$ & $4.3 \%$ \\
\hline Wound infection & $\begin{array}{l}\text { superficial and deep wound infections, organ/space } \\
\text { infections, or wound disruptions }\end{array}$ & $14.2 \%$ & $13.6 \%$ \\
\hline Urinary tract morbidity & $\begin{array}{l}\text { urinary tract infections, progressive renal insufficiency, and } \\
\text { acute renal failure }\end{array}$ & $4.8 \%$ & $5.2 \%$ \\
\hline Central nervous system morbidity & $\begin{array}{l}\text { stroke, coma greater than } 24 \mathrm{hr} \text { postoperatively, and } \\
\text { peripheral nerve injury }\end{array}$ & $0.5 \%$ & $0.2 \%$ \\
\hline Thrombotic morbidity & $\begin{array}{l}\text { pulmonary embolism, deep venous thrombosis, and } \\
\text { thrombophlebitis }\end{array}$ & $2.0 \%$ & $2.2 \%$ \\
\hline Cardiovascular morbidity & $\begin{array}{l}\text { acute myocardial infarction and cardiac arrest requiring } \\
\text { resuscitation }\end{array}$ & $1.0 \%$ & $0.9 \%$ \\
\hline Other & failure of a graft, prosthesis, or flap & $0.1 \%$ & $0.1 \%$ \\
\hline Collapsed composite & Any of the above outcomes ( $v s$ none) & $23.4 \%$ & $22.5 \%$ \\
\hline
\end{tabular}


Table 3 Summary of baseline factors among patients before and after propensity matching. Data are presented as a percentage, mean (standard deviation), or median [1st quartile, 3rd quartile]

\begin{tabular}{|c|c|c|c|c|c|c|}
\hline \multirow[t]{3}{*}{ Factor } & \multicolumn{3}{|c|}{ All Cancer Patients $(n=49,103)$} & \multicolumn{3}{|c|}{ Matched Patients $(n=2,696)$} \\
\hline & \multicolumn{2}{|l|}{ Chemotherapy } & \multirow[b]{2}{*}{ ASD } & \multicolumn{2}{|l|}{ Chemotherapy } & \multirow[b]{2}{*}{ ASD } \\
\hline & No $(n=47,499)$ & Yes $(n=1,604)$ & & No $(n=1,348)$ & Yes $(n=1,348)$ & \\
\hline Female & 59 & 59 & $<0.01$ & 59 & 60 & 0.02 \\
\hline Race & & & 0.08 & & & 0.09 \\
\hline Hispanic & 4 & 5 & & 5 & 5 & \\
\hline Black & 9 & 8 & & 9 & 8 & \\
\hline White & 76 & 76 & & 75 & 76 & \\
\hline Asian or Pacific Islander & 3 & 3 & & 4 & 3 & \\
\hline Unknown/other & 9 & 8 & & 7 & 8 & \\
\hline Body mass index $\left(\mathrm{kg} \bullet \mathrm{m}^{-2}\right)$ & $27[23,31]$ & $27[23,31]$ & 0.09 & $27[23,31]$ & $27[23,31]$ & 0.02 \\
\hline Age & $62(14)$ & $57(13)$ & 0.33 & $58(14)$ & $58(12)$ & 0.03 \\
\hline History of COPD & 5 & 3 & 0.11 & 2 & 3 & 0.05 \\
\hline Type of cancer & & & 0.59 & & & 0.00 \\
\hline Breast cancer & 16 & 24 & & 25 & 25 & \\
\hline Colon cancer & 51 & 35 & & 33 & 33 & \\
\hline Rectal cancer & 4 & 9 & & 9 & 9 & \\
\hline Liver cancer & 11 & 24 & & 25 & 25 & \\
\hline Pancreatic cancer & 18 & 8 & & 8 & 8 & \\
\hline Disseminated cancer & 8 & 37 & 0.74 & 33 & 32 & 0.02 \\
\hline General anesthesia ( $v s$ other) & 98 & 99 & 0.08 & 100 & 99 & 0.07 \\
\hline Dyspnea & 10 & 10 & $<0.01$ & 10 & 10 & 0.01 \\
\hline Hypertension & 49 & 38 & 0.23 & 39 & 40 & 0.02 \\
\hline Renal failure & 0 & 0 & 0.02 & 0 & 0 & 0.02 \\
\hline Diabetes mellitus & & & 0.09 & & & 0.04 \\
\hline Insulin-dependent & 5 & 4 & & 86 & 87 & \\
\hline Orally-controlled & 10 & 8 & & 9 & 9 & \\
\hline None & 85 & 88 & & 5 & 4 & \\
\hline Current smoker (within one year) & 19 & 18 & 0.02 & 18 & 18 & 0.00 \\
\hline Alcohol consumption ( $>2$ drinks/day within 2 weeks) & 3 & 2 & 0.06 & 3 & 2 & 0.04 \\
\hline ASA classification & & & 0.18 & & & 0.04 \\
\hline Healthy & 4 & 2 & & 2 & 2 & \\
\hline Mild disease & 45 & 40 & & 42 & 42 & \\
\hline Severe disease & 47 & 53 & & 53 & 52 & \\
\hline Life-threatening disease & 4 & 5 & & 4 & 4 & \\
\hline Emergency case & 6 & 5 & 0.01 & 5 & 4 & 0.04 \\
\hline Congestive heart failure & 1 & 0 & 0.06 & 0 & 0 & 0.00 \\
\hline Hemiplegia & 0 & 1 & 0.02 & 0 & 1 & 0.02 \\
\hline Stroke & 1 & 1 & 0.01 & 2 & 1 & 0.02 \\
\hline Bleeding disorders & 4 & 4 & 0.04 & 4 & 4 & 0.01 \\
\hline Transfusion $>4$ units before surgery & 0 & 0 & $<0.01$ & 0 & 0 & 0.00 \\
\hline Radiotherapy & 3 & 22 & 0.59 & 15 & 17 & 0.06 \\
\hline Hematocrit $(\%)^{*}$ & $38(5)$ & $37(5)$ & 0.34 & $38(5)$ & $37(5)$ & 0.29 \\
\hline Platelet count $\left(10^{3} \text { platelets } \bullet \mathrm{mL}^{-1}\right)^{*}$ & $260[211,320]$ & $239[184,308]$ & 0.23 & $251[204,308]$ & $241[186,309]$ & 0.11 \\
\hline PTT $(\mathrm{sec})^{*}$ & $29[26,32]$ & $28[26,31]$ & 0.08 & $29[26,32]$ & $29[26,31]$ & 0.06 \\
\hline
\end{tabular}


Table 3 continued

\begin{tabular}{|c|c|c|c|c|c|c|}
\hline \multirow[t]{3}{*}{ Factor } & \multicolumn{3}{|c|}{ All Cancer Patients $(n=49,103)$} & \multicolumn{3}{|c|}{ Matched Patients $(n=2,696)$} \\
\hline & \multicolumn{2}{|l|}{ Chemotherapy } & \multirow[b]{2}{*}{ ASD } & \multicolumn{2}{|l|}{ Chemotherapy } & \multirow[b]{2}{*}{ ASD } \\
\hline & No $(n=47,499)$ & Yes $(n=1,604)$ & & No $(n=1,348)$ & Yes $(n=1,348)$ & \\
\hline International normalized ratio of $\mathrm{PT}^{*}$ & $1[1,1]$ & $1[1,1]$ & 0.04 & $1[1,1]$ & $1[1,1]$ & 0.04 \\
\hline
\end{tabular}

*These preoperative labs were not included in matching due to large amounts of missing data (data were missing for $4.9 \%, 5.9 \%, 44.3 \%$, and $37.5 \%$ of patients, respectively)

ASA = American Society of Anesthesiologists; ASD = absolute standardized difference (absolute difference in means or proportions divided by the pooled standard deviation); $\mathrm{COPD}=$ chronic obstructive pulmonary disease; $\mathrm{PTT}=$ partial thromboplastin time; $\mathrm{PT}=$ prothrombin time

We used R statistical software version 2.13.0 (The R Foundation for Statistical Computing, Vienna, Austria) and SAS ${ }^{\circledR}$ statistical software version 9.2 (SAS Institute, Inc., Cary, NC, USA) for all statistical analyses.

\section{Results}

Among the 971,455 surgical cases available in the ACSNSQIP database, 49,103 were ultimately available for use in our study after a series of inclusions and exclusions, which are summarized in the Figure. Only a tiny proportion of patients were classified as functionally dependent and thus excluded from analysis; we therefore assumed that the 259 patients in whom functional status was unavailable were functionally independent.

In our crude unadjusted analysis, we found that chemotherapy was associated with 30 -day mortality $(\mathrm{OR}=1.71$; 95\% CI 1.21 to $2.42 ; P<0.001)$. Our collapsed composite outcome, on the other hand, did not differ significantly $(\mathrm{OR}=1.07 ; 95 \%$ CI 0.93 to $1.22 ; P=0.825)$. However, these unadjusted results were likely confounded by imbalances in important baseline variables (Table 3). For example, it was more likely that chemotherapy recipients would have disseminated cancer, would be younger, would not have hypertension, would have had radiotherapy, and would have breast, rectal, or liver cancer.

We successfully matched 1,348 pairs of chemotherapy recipients and non-recipients. A summary of baseline demographic and perioperative factors for all patients and matched patients can be found in Table 3. As the small ASD values show (no ASD values $>0.1$ ), the propensity matched patients displayed a much better balance in baseline variables, indicating that propensity matching was successful in limiting the effects of these possible confounders on our analysis.

Postoperative 30-day outcomes for matched patients are summarized in Table 5. Twenty-one of the 1,348 (1.6\%) non-chemotherapy patients died within 30 days after surgery, compared with 30 of the 1,348 (2.2\%) chemotherapy patients. Based on our logistic regression model, the
Table 4 Thirty-day major morbidities and their weights (used for the vector of weighted outcomes) as determined by averaging the scores of three independent anesthesiologists

\begin{tabular}{lc}
\hline Major morbidity & Severity Weight $(\%)$ \\
\hline Superficial SSI & 20 \\
Urinary tract infection & 23 \\
Peripheral nerve injury & 27 \\
Wound disruption & 27 \\
DVT/Thrombosis & 30 \\
Deep SSI & 33 \\
Unplanned tracheal intubation & 37 \\
Failure of a graft, prosthesis, or flap & 37 \\
Organ/Space SSI & 43 \\
Failure to wean & 47 \\
Progressive renal insufficiency & 47 \\
Pneumonia & 47 \\
Pulmonary embolism & 50 \\
Acute renal failure & 50 \\
Stroke & 63 \\
Sepsis & 63 \\
Acute MI & 67 \\
Coma & 70 \\
Septic shock & 80 \\
Cardiac arrest & 80 \\
Mortality & 100 \\
\hline
\end{tabular}

SSI = surgical site infection; $\mathrm{DVT}=$ deep venous thrombosis; $\mathrm{MI}=$ myocardial infarction

corresponding OR $(95 \% \mathrm{CI})$ for mortality (chemotherapy $v s$ non-chemotherapy) was estimated at 1.47 (0.82 to 2.64$)$, which was not statistically significant $(P=0.19)$. Therefore we were unable to conclude that these two groups differed in their risk for mortality.

There was similarly no apparent difference between groups for the collapsed composite outcome $(\mathrm{OR}=1.17$; 95\% CI 0.97 to $1.42 ; P=0.09)$. In our common effect GEE analysis, the severity-weighted vector of outcomes yielded results that were similar to those of the collapsed 


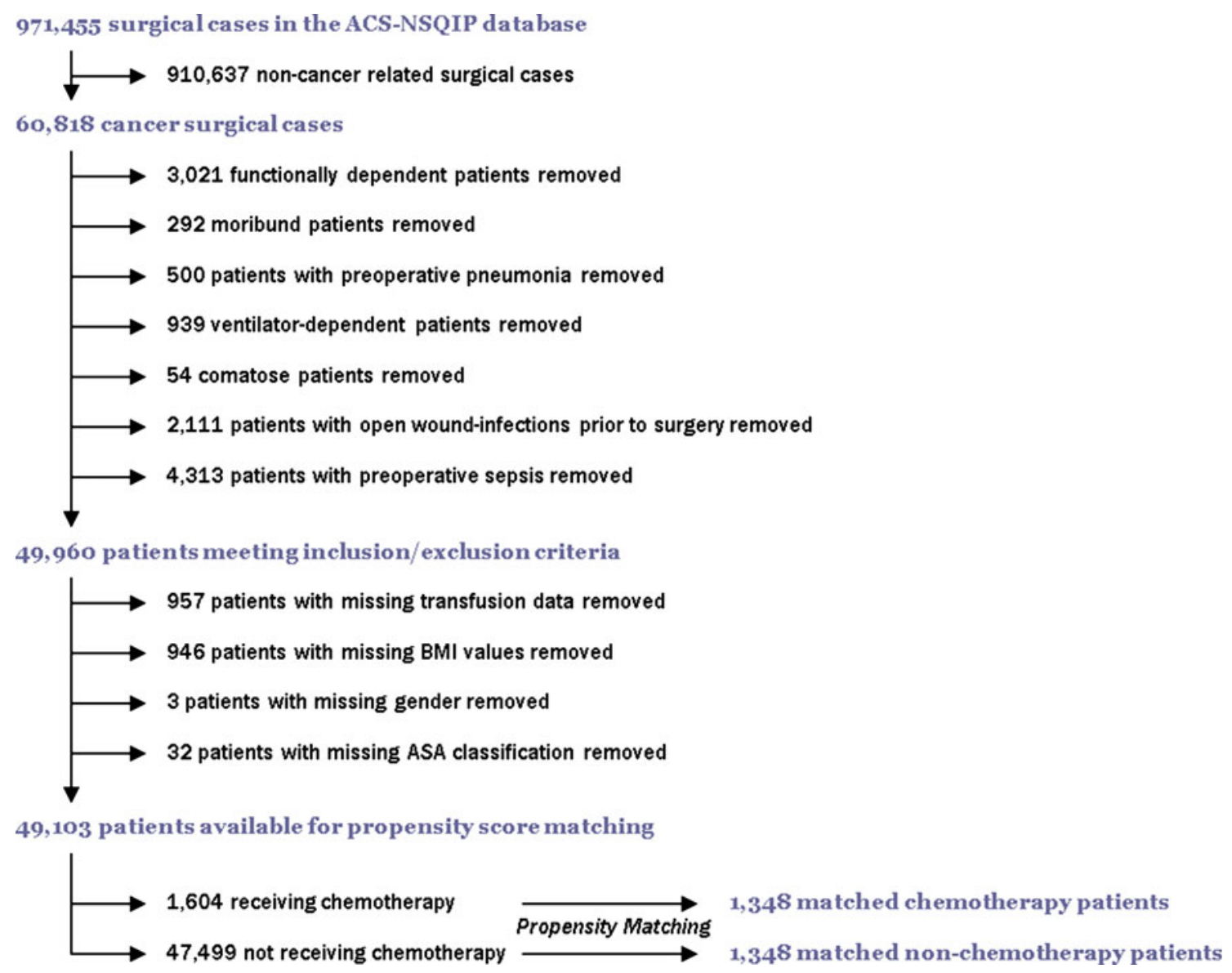

Figure Summary of study inclusion/exclusion criteria and results of matching procedure

composite outcome $(P=0.22)$. The OR $(95 \% \mathrm{CI})$ estimating the global association between chemotherapy and major morbidity weighted by clinical severity of components was 1.15 (0.92 to 1.43 ).

\section{Discussion}

Our analysis indicates that the odds of mortality or composite morbidity 30 days after surgery appears not to differ in patients who were or were not given chemotherapy within 30 days before surgery. This result is surprising since all chemotherapeutic agents, regardless of type, produce a wide range of detrimental effects. For example, common chemotherapeutic drugs cause immune suppression, pulmonary and cardiac toxicity, and myelosuppression. Our results are consistent with some previous studies but diverge from others.

For example, some studies suggest that chemotherapy is a perioperative risk factor while other studies failed to show any association between neoadjuvant chemotherapy and perioperative morbidity or mortality. ${ }^{9,17}$ Two other studies identified an increase in infectious complications and delayed wound healing with chemotherapy. ${ }^{18,19} \mathrm{We}$ note though that the infection studies were restricted to patients having surgery for rectal cancer, which has an extremely high baseline infection risk. In contrast, we included a broad range of surgical indications.

Meric et al. ${ }^{9}$ found no association between chemotherapy and mortality and morbidity, which is consistent with our findings. In two other studies, there was also no association with chemotherapy and mortality or morbidity in patients having surgery for esophageal cancer. Our results are also consistent with Shapiro et $a .^{20}$ who did not find any intraoperative or postoperative complications in breast cancer patients given doxorubicin before surgery. A potential limitation of studies that did not identify an adverse effect of preoperative chemotherapy is that most were small and therefore possibly underpowered to detect important clinical outcomes. Our analysis of 2,696 matched patients is a much larger study to evaluate the potential adverse effects of preoperative chemotherapy. While our matched sample contained a sufficient number of events for detecting clinically meaningful differences in the composite outcome, the low incidence of mortality resulted in rather imprecise estimates of the effect of 
Table 5 Association between chemotherapy status and major morbidity outcomes, including the composite outcome, before and after propensity matching

\begin{tabular}{|c|c|c|c|c|}
\hline \multirow[t]{3}{*}{ Outcome } & \multicolumn{4}{|c|}{ Matched Patients $(n=2,696)$} \\
\hline & \multicolumn{2}{|l|}{ Chemotherapy } & \multirow[b]{2}{*}{ OR $(95 \% \mathrm{CI})$} & \multirow[b]{2}{*}{$P$ value } \\
\hline & No $(n=1,348) n(\%)$ & Yes $(n=1,348) n(\%)$ & & \\
\hline 30-day mortality & $21(1.6)$ & $30(2.2)$ & 1.47 (0.82 to 2.64$)$ & 0.19 \\
\hline Collapsed composite of major morbidities* & $286(21.2)$ & $321(23.8)$ & $1.17(0.97$ to 1.42$)$ & 0.09 \\
\hline Weighted vector of major morbidities $\dagger$ & - & - & $1.15(0.92$ to 1.43$)$ & 0.22 \\
\hline \multicolumn{5}{|l|}{ Individual morbidities } \\
\hline Systemic infections & $88(6.5)$ & $101(7.5)$ & - & - \\
\hline Respiratory & $57(4.2)$ & $59(4.4)$ & - & - \\
\hline Wound infection & $176(13.1)$ & $191(14.2)$ & - & - \\
\hline Urinary tract & $63(4.7)$ & $77(5.7)$ & - & - \\
\hline Central Nervous System & $3(0.2)$ & $2(0.1)$ & - & - \\
\hline Thrombotic & $26(1.9)$ & $33(2.4)$ & - & - \\
\hline Cardiovascular & $11(0.8)$ & $12(0.9)$ & - & - \\
\hline Other & $0(0.0)$ & $2(0.1)$ & - & - \\
\hline
\end{tabular}

*The collapsed composite is defined as any ( $v s$ none) of the major morbidities listed in Table 1

$\dagger$ The weighted vector of major morbidities used severity weights (as determined by three independent anesthesiologists) of each major morbidity in a multivariable generalized estimating equations (GEE) model to estimate the odds ratio, which is the estimated global association between chemotherapy and major morbidity weighted by the severity of its components

$\mathrm{CI}=$ confidence interval

chemotherapy on postoperative mortality (we had 51 deaths in our matched sample).

We exactly matched for type of surgery, which is a major determinant of early postoperative outcome. We similarly adjusted for all available imbalanced and preoperative baseline factors that may affect short-term perioperative outcomes. Nonetheless, our retrospective analysis was limited by availability of data in the ACSNSQIP registry, which lacked important potential confounding variables, such as the type and staging of the cancer and the type of chemotherapy. The extent to which these and other potential confounding factors may have influenced our conclusions is unclear, but presumably, chemotherapy was more likely to have been used for advanced disease and thus in patients at greatest perioperative risk. Even considering this potential bias, our data did not suggest an increase in mortality or major morbidities in patients given preoperative chemotherapy. There are some other limitations of the ACS-NSQIP registry. The ACS-NSQIP is limited to the hospitals participating, which may not be a valid national representative sample of hospitals, although ACS-NSQIP is by far the largest available surgical registry. Furthermore, not all the cases are entered from participating hospitals; however, any bias relating to the chemotherapy status of patients entered in the registry is unlikely.

Our analysis was restricted to 30-day morbidities and mortality. We thus cannot estimate the longer-term (i.e., one-year) consequences of preoperative chemotherapy; however, since 30-day morbidity and mortality were comparable with and without chemotherapy, it appears that the acute effects are not harmful - leaving open the possibility of long-term benefit in terms of reducing the risk of cancer recurrence.

In summary, we found no association between the preoperative use of neoadjuvant chemotherapy and early postoperative complications or mortality in cancer patients undergoing resection surgeries. In cases where preoperative chemotherapy potentially reduces the risk of local or metastatic recurrence, our results suggest that it should generally not be avoided for fear of acute perioperative toxicity.

Conflicts of interest None declared.

\section{References}

1. Kochanek KD, Xu J, Murphy SL, Minino AM, Kung HC. Deaths: Preliminary data for 2009, National Vital Statistics Reports. Hyattsville, MD, National Center for Health Statistics, 2011. Available from URL: http://www.cdc.gov/nchs/data/nvsr/nvsr59/ nvsr59_04.pdf (accessed April 2012)

2. Leong T, Guiney M, Ngan S, Mackay J. Pre-operative radiotherapy and chemotherapy for non-resectable rectal cancer. Aust N Z J Surg 1997; 67: 603-6.

3. Nygaard $K$, Hagen $S$, Hansen $H S$, et al. Pre-operative radiotherapy prolongs survival in operable esophageal carcinoma: a 
randomized, multicenter study of pre-operative radiotherapy and chemotherapy. The second Scandinavian trial in esophageal cancer. World J Surg 1992; 16: 1104-9.

4. Kaklamanos IG, Walker GR, Ferry K, Franceschi D, Livingstone $A S$. Neoadjuvant treatment for resectable cancer of the esophagus and the gastroesophageal junction: a meta-analysis of randomized clinical trials. Ann Surg Oncol 2003; 10: 754-61.

5. Urschel JD, Ashiku S, Thurer R, Sellke FW. Salvage or planned esophagectomy after chemoradiation therapy for locally advanced esophageal cancer-a review. Dis Esophagus 2003; 16: 60-5.

6. Pisters KM, Vallieres E, Crowley JJ, et al. Surgery with or without preoperative paclitaxel and carboplatin in early-stage non-small-cell lung cancer: Southwest Oncology Group Trial S9900, an intergroup, randomized, phase III trial. J Clin Oncol 2010; 28: 1843-9.

7. Nordlinger B, Sorbye $H$, Glimelius $B$, et al. Perioperative chemotherapy with FOLFOX4 and surgery versus surgery alone for resectable liver metastases from colorectal cancer (EORTC Intergroup trial 40983): a randomised controlled trial. Lancet 2008; 371: 1007-16.

8. Tabira Y, Okuma T, Kondo K, et al. Does neoadjuvant chemotherapy for carcinoma in the thoracic esophagus increase postoperative morbidity? Jpn J Thorac Cardiovasc Surg 1999; 47: 361-7.

9. Meric $F$, Milas $M$, Hunt KK, et al. Impact of neoadjuvant chemotherapy on postoperative morbidity in soft tissue sarcomas. J Clin Oncol 2000; 18: 3378-83.

10. Malthaner RA, Collin S, Fenlon D. Preoperative chemotherapy for resectable thoracic esophageal cancer. Cochrane Database Syst Rev 2006; 3: CD001556.

11. Dahn D, Martell J, Vorwerk H, et al. Influence of irradiated lung volumes on perioperative morbidity and mortality in patients after neoadjuvant radiochemotherapy for esophageal cancer. Int $\mathbf{J}$ Radiat Oncol Biol Phys 2010; 77: 44-52.

12. Kesmodel SB, Ellis LM, Lin E, et al. Preoperative bevacizumab does not significantly increase postoperative complication rates in patients undergoing hepatic surgery for colorectal cancer liver metastases. J Clin Oncol 2008; 26: 5254-60.

13. Fink AS, Campbell DA Jr, Mentzer RM Jr, et al. The National Surgical Quality Improvement Program in non-veterans administration hospitals: initial demonstration of feasibility. Ann Surg 2002; 236: 344-54.

14. Khuri SF. The NSQIP: a new frontier in surgery. Surgery 2005; 138: 837-43.

15. Normand ST, Landrum MB, Guadagnoli E, et al. Validating recommendations for coronary angiography following acute myocardial infarction in the elderly: a matched analysis using propensity scores. J Clin Epidemiol 2001; 54: 387-98.

16. Cohen J. Statistica Power Analysis for the Behavioral Sciences. 2nd ed. Hillsdale: Lawrence Erlbaum Associates; 1988.

17. Kelley ST, Coppola D, Karl RC. Neoadjuvant chemoradiotherapy is not associated with a higher complication rate vs. surgery alone in patients undergoing esophagectomy. J Gastrointest Surg 2004; 8: 227-31.

18. Buie WD, MacLean AR, Attard JA, Brasher PM, Chan AK. Neoadjuvant chemoradiation increases the risk of pelvic sepsis after radical excision of rectal cancer. Dis Colon Rectum 2005; 48: 1868-74.

19. Artioukh DY, Smith RA, Gokul K. Risk factors for impaired healing of the perineal wound after abdominoperineal resection of rectum for carcinoma. Colorectal Dis 2007; 9: 362-7.

20. Shapiro $R$, Barsuk D, Segev L, et al. Pre-operative cardiac workup after anthracycline-based neoadjuvant chemotherapy. Is it really necessary? Ann R Coll Surg Engl 2011; 93: 127-9. 